\title{
Partial Information Relaying with Per Antenna Superposition Coding
}

\author{
Dong In Kim, Wan Choi, Hanbyul Seo, and Byoung-Hoon Kim
}

\begin{abstract}
In this letter we propose per antenna superposition coding (PASC) by which partial information can be relayed instead of full information, to exploit the higher capacity of source-relay-destination link. Here, the PASC is designed across antennas, producing basic layer and superposed layer for each data stream per antenna. It is shown that an overall data rate of partial information relaying with PASC can be increased beyond that offered by full information relaying by virtue of fast forwarding of partial information over relatively better link.
\end{abstract}

Index Terms-Cooperative relaying, multiple antennas, per antenna superposition coding (PASC), partial information, rate matching, power allocation.

\section{INTRODUCTION}

$\mathbf{T}$ HERE have been lots of efforts to improve the capacity of direct transmission via cooperative relaying when a user experiences weaker channel condition. Especially, it is crucial in cellular environments to mitigate intercell interference for an increased rate and extended coverage at cell edge. In such efforts, various relaying protocols such as amplify-and-forward (AF), decode-and-forward (DF), compress-and-forward (CF) have been proposed [1], [2], [3], [4]. Those relaying schemes are mainly to exploit the multipath diversity for power gain (or increased rate) that results from combining direct and relayed signals. However, they do not fully utilize the asymmetric link capacities existing in direct and relay links, where the latter is typically in better condition when line-of-sight (LOS) transmission is realized for a fixed relay.

In order to more efficiently exploit the asymmetric link capacities, partial decode-and-forward protocol was first proposed in [5], where a relay forwards part of decoded information to a destination based on superposition coding. Recently, Popovski and de Carvalho [6] investigated the power division between basic data and superposed data for overall rate maximization. However, the multiple antenna configurations that cause additional inter-stream interference due to spatial multiplexing were not considered in their work, which is prerequisite for realization of next-generation cellular systems.

The concept of partial DF relaying scheme can be translated into multiple antenna configurations by applying superposition

This research was supported by the MKE (Ministry of Knowledge Economy), Korea, under the ITRC (Information Technology Research Center) support program supervised by the NIPA (National IT Industry Promotion Agency) (NIPA-2010-(C1090-1011-0005)) and LG Electronics, Inc.

Dong In Kim is with the School of Information and Communication Engineering, Sungkyunkwan University (SKKU), Suwon 440-746, Korea (Email: dikim@ece.skku.ac.kr). Wan Choi is with the Department of Electrical Engineering, KAIST, Daejeon, Korea (E-mail: wchoi@ee.kaist.ac.kr). Hanbyul Seo and Byoung-Hoon Kim are with LG Electronics, Inc. (E-mail: \{hbseo, byoungk\}@lge.com). coding per antenna, termed per antenna superposition coding $(P A S C)$. But the extension is not straightforward because the power should be divided not only between the basic and superposed layers but also across spatial layers. Furthermore, the power division interacts with spatial interference.

In this context, this letter aims to find a proper power division strategy for partial DF relaying with PASC and to eventually show how another form of relaying based on PASC can be effective in increasing an overall data rate at cell edge when multiple antennas are employed.

The rest of this letter is organized as follows. Section II describes system and channel models for relaying with PASC. An optimal power allocation between the two layers and across antennas is performed to maximize an overall data rate in Section III. The achieved capacity of the proposed partial DF relaying with PASC is evaluated and compared with those of conventional relaying schemes in Section IV. Conclusions are drawn in Section V.

\section{Partial Information Relaying with Per ANTENNA SUPERPOSITION CODING}

We consider a multiple antenna configuration of $M$ transmit and $N$ receive antennas at a source and a destination, respectively. A relay operates in half duplexing mode and has $N$ receive and $N$ transmit antennas. We assume that the slot length in the first hop and second hop adaptively varies according to the amount of information to be transferred and the link conditions for efficient link utilization. Also, we assume a quasi-static channel over two hops, over which the link conditions remain unchanged. If this assumption is not feasible in practice, we need some form of prediction (for details refer to [7]) based on the feedback about link conditions for joint power allocation between source and relay in Section III. The transmit signal vector at a source is

$$
\mathbf{x}=\left[x_{1}, x_{2}, \ldots, x_{M}\right]^{T}
$$

where $x_{m}=\sqrt{1-\alpha_{m}} x_{b, m}+\sqrt{\alpha_{m}} x_{s, m},\left\{\alpha_{m}\right\}$ represent the power division factors between the basic and superposed layers of each data stream per antenna, the power allocation to antenna $m$ is $P_{m}=E\left[\left|x_{b, m}\right|^{2}\right]=E\left[\left|x_{s, m}\right|^{2}\right],(\cdot)^{T}$ and $E[\cdot]$ denote transpose and expectation, respectively. Note that the subscript $b$ denotes the basic layer while $s$ indicates the superposed layer on top of basic layer.

If both path loss and fading are accounted for, the $N \times 1$ received signal vector at a destination is of the form

$$
\mathbf{y}_{0}=\sqrt{\mu_{0}} \mathbf{H}_{0} \mathbf{x}+\mathbf{n}_{0}
$$


where $\mu_{0}$ is the channel attenuation due to path loss, the $N \times M$ channel matrix of direct link $\mathbf{H}_{0}$ is composed of independent zero-mean complex Gaussian random variables (flat fading), and $\mathbf{n}_{0}$ denotes the zero-mean additive white Gaussian noise (AWGN) vector with $E\left[\mathbf{n}_{0} \mathbf{n}_{0}^{H}\right]=\sigma^{2} \mathbf{I}_{N},(\cdot)^{H}$ and $\mathbf{I}_{N}$ denote Hermitian transpose and the identity matrix of size $N \times N$, respectively.

Similarly, the $N \times 1$ received signal vector at a relay can be formulated as

$$
\mathbf{y}_{1}=\sqrt{\mu_{1}} \mathbf{H}_{1} \mathbf{x}+\mathbf{n}_{1}
$$

where the channel and noise statistics associated with different links and antennas are assumed statistically independent. Note that no precoding across multiple transmit antennas at both source and relay is considered because we assume the feedback about per antenna post detection signal-to-interferenceplus-noise (SINR) instead of full channel state information (CSI).

At the relay, zero-forcing (ZF) V-BLAST [8] is employed for the successive interference cancellation and spatial decorrelation of the inter-stream interference. The ZF-nulling vector for the stream to be decoded in the $j$ th order is given by

$$
\mathbf{w}_{1, l_{j}}=\left(\mathbf{H}_{1, \bar{l}_{j-1}}^{+}\right)_{l_{j}}^{T}
$$

where $l_{j}$ is the index of the stream to be decoded in the $j$ th order, $\mathbf{H}_{1, \bar{l}_{j-1}}$ is the matrix obtained by zeroing columns $l_{1}, l_{2}, \ldots, l_{j-1}$ of $\mathbf{H}_{1},(\cdot)^{+}$denotes the Moore-Penrose pseudoinverse [9], and $(\cdot)_{j}$ denotes the $l_{j}$ th row of given matrix. Note that the $j$ th decoding order is determined by

$$
l_{j}=\operatorname{argmin}_{n \notin l_{1}, \ldots, l_{j-1}}\left\|\left(\mathbf{H}_{1, \bar{l}_{j-1}}^{+}\right)_{n}\right\|^{2} .
$$

After spatial decorrelation by the ZF-nulling vector, the basic data is decoded first and then the superposed data is decoded by subtracting off the effects of the basic data. Assuming that the data stream through antenna $m$ is decoded at the relay in the $j$ th order, the post detection SINRs of basic and superposed data on the $m$ th antenna are given, respectively, by

$$
\begin{aligned}
\rho_{b, m}^{1} & =\frac{\left(1-\alpha_{m}\right) \gamma_{1, m}}{\left\|\mathbf{w}_{1, l_{j}}\right\|^{2}+\alpha_{m} \gamma_{1, m}} \\
\rho_{s, m}^{1} & =\frac{\alpha_{m} \gamma_{1, m}}{\left\|\mathbf{w}_{1, l_{j}}\right\|^{2}}
\end{aligned}
$$

where the superscript 1 denotes the relay and $\gamma_{1, m}$ is the per antenna link SNR from source to relay and given by $\gamma_{1, m}=$ $\mu_{1} P_{m} / \sigma^{2}$.

In the second hop, the relay forwards only the superposed layers. In the adaptive slot length protocol, the duration of the second hop can be much shorter than that of the first hop since only partial information need to be transferred over relatively better link. This might contribute to an increase in overall rate by virtue of the fast forwarding of partial information. Once the destination successfully decodes the superposed layers, where sufficient energy for successful decoding can be accumulated by variable duration in the second hop, the effects of superposed layers are cancelled out from the signal received in the first hop. Consequently, the basic layers become free from the interference by superposed layers. It should be noted that enabling the destination to receive data in the first hop is critical to the system performance, as emphasized in the previous works [10] and [11]. Applying ZF V-BLAST, the ZF-nulling vector for the stream to be decoded in the $i$ th order is given by

$$
\mathbf{w}_{0, k_{i}}=\left(\mathbf{H}_{0, \bar{k}_{i-1}}^{+}\right)_{k_{i}}^{T}
$$

where $k_{i}$ is the index of the stream to be decoded in the $i$ th order and determined by

$$
k_{i}=\operatorname{argmin}_{n \notin k_{1}, \ldots, k_{i-1}}\left\|\left(\mathbf{H}_{0, \bar{k}_{i-1}}^{+}\right)_{n}\right\|^{2} .
$$

Assuming that the data stream through antenna $m$ is decoded at the destination in the $i$ th order, the post detection SINR of basic layer on the $m$ th antenna is given by

$$
\rho_{b, m}^{0}=\frac{\left(1-\alpha_{m}\right) \gamma_{0, m}}{\left\|\mathbf{w}_{0, k_{i}}\right\|^{2}}
$$

where the superscript 0 denotes the destination and $\gamma_{1, m}$ is the per antenna link SNR from source to destination and given by $\gamma_{0, m}=\mu_{0} P_{m} / \sigma^{2}$.

\section{Optimal Power And Rate Allocation}

Now, the achievable rates for basic layer and superposed layer through antenna $m$ are determined by

$$
\begin{aligned}
& R_{b, m}=\log \left(1+\rho_{b, m}\right) \\
& R_{s, m}=\log \left(1+\rho_{s, m}\right)
\end{aligned}
$$

where $\rho_{b, m}=\min \left(\rho_{b, m}^{0}, \rho_{b, m}^{1}\right)$ and $\rho_{s, m}=\rho_{s, m}^{1}$. Since we are assuming the feedback from destination and relay to source about per antenna post detection SINRs, an optimal power and rate allocation between the basic and superposed layers and across antennas is attempted to maximize an overall data rate. That is, the overall rate offered by partial information relaying with multiple antennas, denoted by $R_{s c}(M, N)$, is maximized by finding an optimum combination of power division factors $\left\{\alpha_{m}\right\}$ and per antenna power allocation $\left\{P_{m}\right\}$.

Reflecting the adaptive slot length protocol, the problem for optimizing the power allocation above can be formulated as

$$
\begin{gathered}
\left(\left\{\alpha_{m}\right\},\left\{P_{m}\right\}\right)=\operatorname{argmax} \underset{\substack{\left\{\alpha_{m}\right\},\left\{P_{m}\right\} \\
\sum_{m=1}^{M} P_{m} \leq P_{T}}}{\arg }\left\{R_{s c}(M, N)=\right. \\
\left.\frac{\sum_{m=1}^{M} R_{b, m}\left(\alpha_{m}, P_{m}\right)+R_{s, m}\left(\alpha_{m}, P_{m}\right)}{1+\sum_{m=1}^{M} R_{s, m}\left(\alpha_{m}, P_{m}\right) / R_{2}(N)}\right\}
\end{gathered}
$$

where $\left(\alpha_{m}, P_{m}\right)$ denote explicitly the dependence of $R_{b, m}$ and $R_{s, m}$ on these parameters. Here, the capacity of relay to destination link (i.e., R-D link) $R_{2}(N)$ is evaluated as

$$
R_{2}(N)=\sum_{n=1}^{N} R_{2, n}=\sum_{n=1}^{N} \log \left(1+\rho_{2, n}\right)
$$

where the post detection SINR at the $n$th receive antenna of the R-D link is

$$
\rho_{2, n}=\frac{\gamma_{2, n}}{\left\|\mathbf{w}_{2, \tilde{l}_{j}}\right\|^{2}}
$$


Here, $\gamma_{2, n}=\mu_{2} \tilde{P}_{n} / \sigma^{2}$ is the R-D link SNR at the $n$th antenna, and $\tilde{P}_{n}$ denotes the power allocation to $n$th antenna of the R$\mathrm{D}$ link. Note that the ZF-nulling vector of the R-D link $\mathbf{w}_{2, \tilde{l}_{j}}$ can be obtained similarly as in (4) with $H_{1}$ replaced by the $\mathrm{N} \times \mathrm{N}$ channel matrix of the R-D link $\mathrm{H}_{2}$.

Note that for given $\left\{P_{m}\right\}$ the overall rate $R_{s c}(M, N)$ is maximized if the rates are matched as $\rho_{b, m}^{0}=\rho_{b, m}^{1}$ because of decorrelation after the projection by each ZF-nulling vector. For the rate matching, i.e., $\rho_{b, m}^{0}=\rho_{b, m}^{1}$, the power division factors should be set to

$$
\alpha_{m}=\left(\frac{\left\|\mathbf{w}_{0, k_{i}}\right\|^{2}}{\gamma_{0, m}}-\frac{\left\|\mathbf{w}_{1, l_{j}}\right\|^{2}}{\gamma_{1, m}}\right)^{+} \quad \text { for } m=1,2, \ldots, M
$$

where $(x)^{+}=\max (0, x)$. Then, the overall capacity offered by partial information relaying with PASC is simplified to

$$
R_{s c}(M, N)=\frac{\sum_{m=1}^{M} \log \left(1+\frac{\gamma_{1, m}}{\left\|\mathbf{w}_{1, l_{j}}\right\|^{2}}\right)}{1+\sum_{m=1}^{M} \theta\left(\alpha_{m}\right) \log \left(\frac{\mu_{1}\left\|\mathbf{w}_{0, k_{i}}\right\|^{2}}{\mu_{0}\left\|\mathbf{w}_{1, l_{j}}\right\|^{2}}\right) / R_{2}(N)}
$$

for $0 \leq \alpha_{m}<1$ where $\theta\left(\alpha_{m}\right)=0$ if $\alpha_{m}=0$ and otherwise one.

If equal power split between source and relay is assumed, and the total power constraints are $\sum_{m=1}^{M} P_{m} \leq P_{T}$ and $\sum_{n=1}^{N} \tilde{P}_{n} \leq P_{T}$, the optimum power allocation is shown to be

$$
\begin{gathered}
P_{m}=\left(\frac{1}{\lambda_{1}}-\frac{\sigma^{2}\left\|\mathbf{w}_{1, l_{j}}\right\|^{2}}{\mu_{1}}\right)^{+} \\
\tilde{P}_{n}=\left(\frac{1}{\lambda_{2}}-\frac{\sigma^{2}\left\|\mathbf{w}_{2, \tilde{l}_{j}}\right\|^{2}}{\mu_{2}}\right)^{+}
\end{gathered}
$$

where $\lambda_{1}$ and $\lambda_{2}$ are determined to meet the total power constraints above.

On the other hand, if unequal power split between the source and relay is assumed under a total power constraint $\sum_{m=1}^{M} P_{m}+\sum_{n=1}^{N} \tilde{P}_{n} \leq 2 P_{T}$, the optimum power allocation can be found through numerical search as follows:

1) Set the total power constraints to be $\sum_{m=1}^{M} P_{m} \leq(1+$ $\epsilon) P_{T}$ and $\sum_{n=1}^{N} \tilde{P}_{n} \leq(1-\epsilon) P_{T}$ with fixed $\epsilon$, subject to the total sum power of $2 P_{T}$.

2) The optimum power allocation can be determined in (16) and (17) by properly choosing $\lambda_{1}$ and $\lambda_{2}$ to meet the corresponding total power constraints for a given $\epsilon$.

3) An efficient search method can be employed to fast track down the search space $|\epsilon|<1$ since the capacity of interest appears to be a concave function of $\epsilon$.

Note that under the perfect rate matching in (14), the capacity of superposed layer over the S-D link remains fixed regardless of the power allocation and is given by

$$
R_{s c}(M, N)=\sum_{m=1}^{M} \theta\left(\alpha_{m}\right) \log \left[\left(\mu_{1}\left\|\mathbf{w}_{0, k_{i}}\right\|^{2}\right) /\left(\mu_{0}\left\|\mathbf{w}_{1, l_{j}}\right\|^{2}\right)\right],
$$

and the overall capacity $R_{s c}(M, N, \epsilon)$ in (15) as a function of $\epsilon$ can be maximized by applying the above total power constraints, respectively.
The resulting maximum capacity $\max _{|\epsilon|<1} R_{s c}(M, N, \epsilon) \triangleq$ $R_{s c, \max }(M, N)$ will be compared with $R_{s c}(M, N, \epsilon=0)$ that is offered by the equal power split in the following Section.

\section{NumericAl Results}

The proposed partial information relaying with PASC is compared in terms of the capacity (bits/symbol) with the following conventional schemes when $M=N=4$ :

- Two-hop transmission: full information decoded at the relay is forwarded to the destination in the second hop. So it corresponds to a special case of the proposed partial information relaying with PASC when $\alpha_{m}=1, \forall m$. The capacity in the adaptive slot length protocol becomes

$$
R_{2 h}=R_{1} /\left(1+R_{1} / R_{2}\right)
$$

where $R_{1}=\sum_{m} \log \left(1+\rho_{b, m}^{1}\right)$ and $R_{2}=\sum_{m} \log (1+$ $\left.\rho_{b, m}^{0}+\rho_{2, m}\right)$. Here $\rho_{b, m}^{1}, \rho_{b, m}^{0}$, and $\rho_{2, m}$ are found in (5), (8), and (13), respectively, when $\alpha_{m}=0, \forall m$. Note that the rate of the R-D link is obtained by the maximalratio combining (MRC) of the signals received at the destination in the first hop and second hop.

- Conventional DF relaying: full information decoded at the relay is forwarded in the second hop, but a fixed slot length is adopted. The capacity is given by

$$
R_{D F}=\frac{1}{2} \min \left(R_{1}, R_{2}\right)
$$

where $R_{1}$ and $R_{2}$ are the same as given in the two-hop transmission.

- Direct transmission: no relay is employed.

Fig. 1 shows the capacities of the proposed partial information relaying with PASC and other conventional schemes when $\gamma_{1}=\mu_{1} P_{T} / \sigma^{2}=15 \mathrm{~dB}$ and $\gamma_{2}=\mu_{2} P_{T} / \sigma^{2}=20$ $\mathrm{dB}$. It is seen that the partial information relaying with PASC outperforms full information relaying, and yields higher capacity than direct transmission $\left(R_{0}\right)$ when $\gamma_{0}=\mu_{0} P_{T} / \sigma^{2}<13$ $\mathrm{dB}$ for equal power split and $\gamma_{0}<16 \mathrm{~dB}$ for unequal power split, which are typical in cellular relay environments. Unequal power split between source and relay further makes the partial information relaying with PASC more attractive than full information relaying and direct transmission.

The capacities for different geometry from Fig. 1 are shown in Fig. 2 when $\gamma_{1}=20 \mathrm{~dB}$ and $\gamma_{2}=15 \mathrm{~dB}$. Compared to Fig. 1, the capacity decrease of the proposed scheme with unequal power split is marginal since unequal power allocation between source and relay helps to compensate for the degradation of the R-D link. On the other hand, the capacity for equal power split notably increases when $\gamma_{0}>10$ $\mathrm{dB}$ since the degraded R-D link can be compensated by the improved S-R link.

Fig. 3 shows the capacity versus $\gamma_{0}$ and the power division factor $\epsilon$ that indicates unequal power split between source and relay, when $\gamma_{1}=20 \mathrm{~dB}$ and $\gamma_{2}=20 \mathrm{~dB}$. An optimal value of $\epsilon$ in general increases as $\gamma_{0}$ increases since better S-D link renders more power allocation for superposed layers. It is also noted that the proposed algorithm can effectively locate the optimal value of $\epsilon$. 


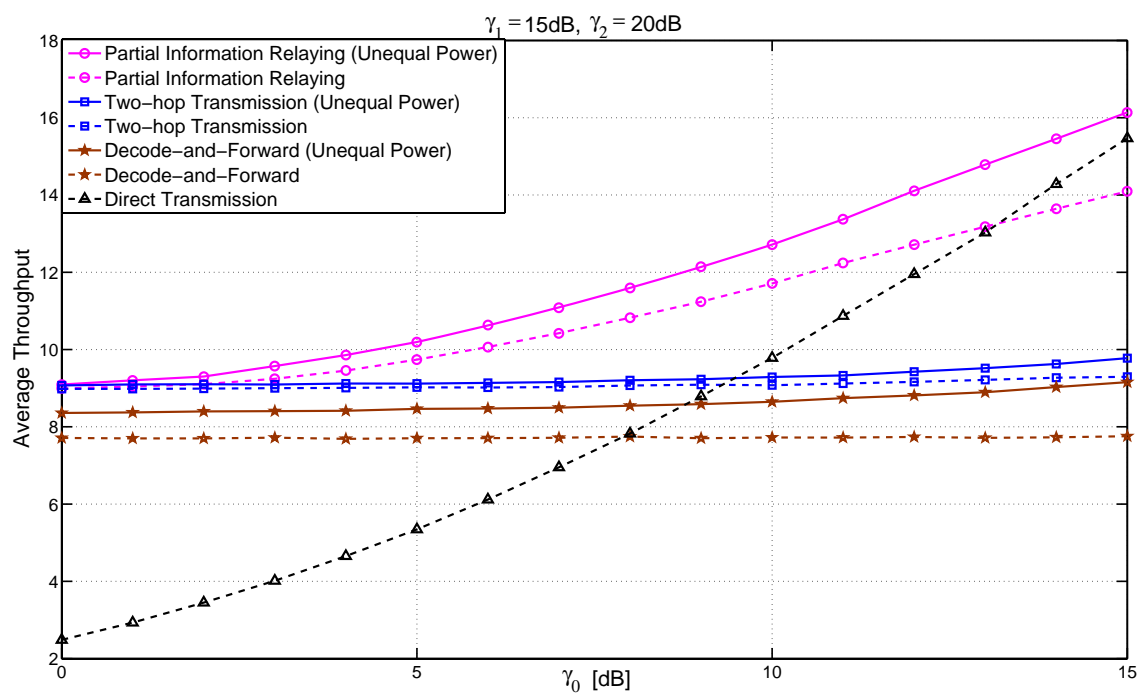

Fig. 1. Capacity (bits/symbol) when $\gamma_{1}=15 \mathrm{~dB}, \gamma_{2}=20 \mathrm{~dB}$, and $M=N=4$.

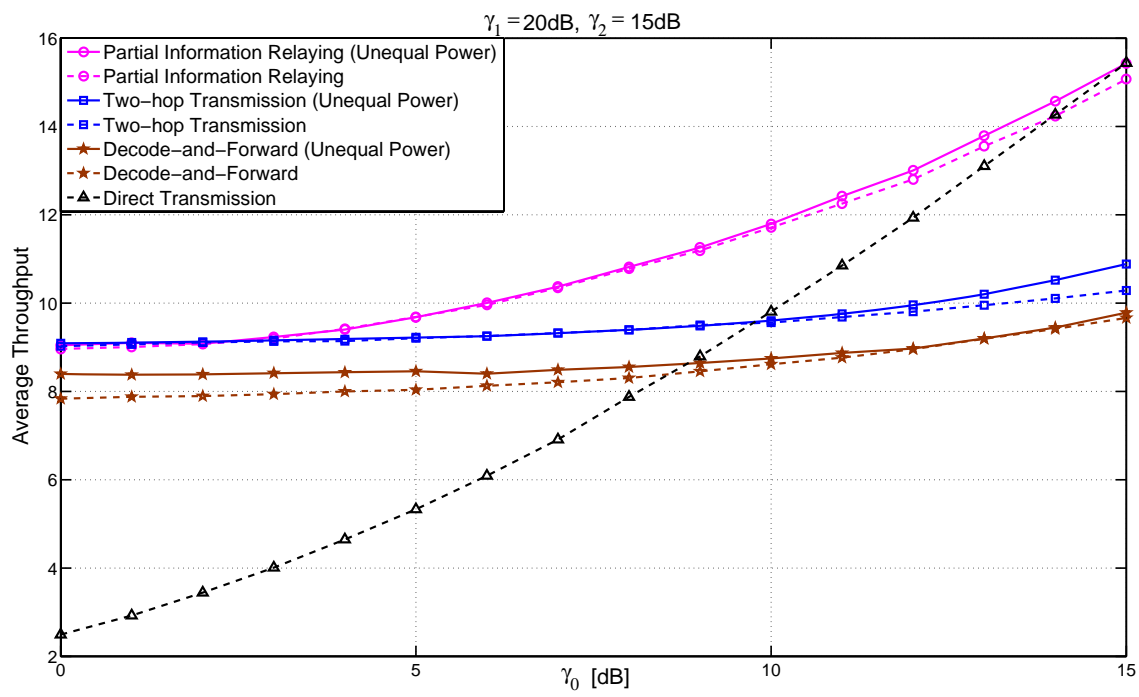

Fig. 2. Capacity (bits/symbol) when $\gamma_{1}=20 \mathrm{~dB}, \gamma_{2}=15 \mathrm{~dB}$, and $M=N=4$.

Fig. 4 depicts average throughput when superposition of two independent QPSK modulated symbols is adopted for the transmission of basic and superposed layers, and $\alpha_{m}=0.2$ is used in (1) to have a standard 16-QAM constellation with unit average power. Following the framework presented in Section $\mathrm{V}$ of [6] and assuming that the 16-QAM transmissions over S-R and R-D links are error free for the given configuration $\left(\gamma_{1}=\gamma_{2}=25 \mathrm{~dB}\right)$, the average throughput for the proposed scheme is

$$
R_{s c, Q P S K}=\frac{2 N M+\sum_{m=1}^{M} 2 N P_{Q P S K}\left[\left(1-\alpha_{m}\right) \gamma_{0, m}\right]}{3 N / 2}
$$

where $N$ is the number of symbols and $P_{Q P S K}(\gamma)$ denotes the probability that the QPSK modulated symbol is correctly received with $\gamma$. It is confirmed that the partial information re- laying with PASC takes benefits even when $\alpha_{m}$ has only three discrete values which determine effective transmission mode among multi-hop transmission $\left(\alpha_{m}=1\right)$, partial information relaying $\left(\alpha_{m}=0.2\right)$, and direct transmission $\left(\alpha_{m}=0\right)$. Note that the proposed relaying with PASC generalizes the three transmission modes by the parameter $\alpha_{m}$.

\section{CONCLUSION}

We have shown that partial information relaying with per antenna superposition coding (PASC) allows for perfect rate matching among asymmetric links often observed at cell edge, resulting in significant capacity gain over full information relaying. In addition, the joint rate and power allocation using the feedback about per antenna post detection SINR was shown to be effective to yield an increased gain for 


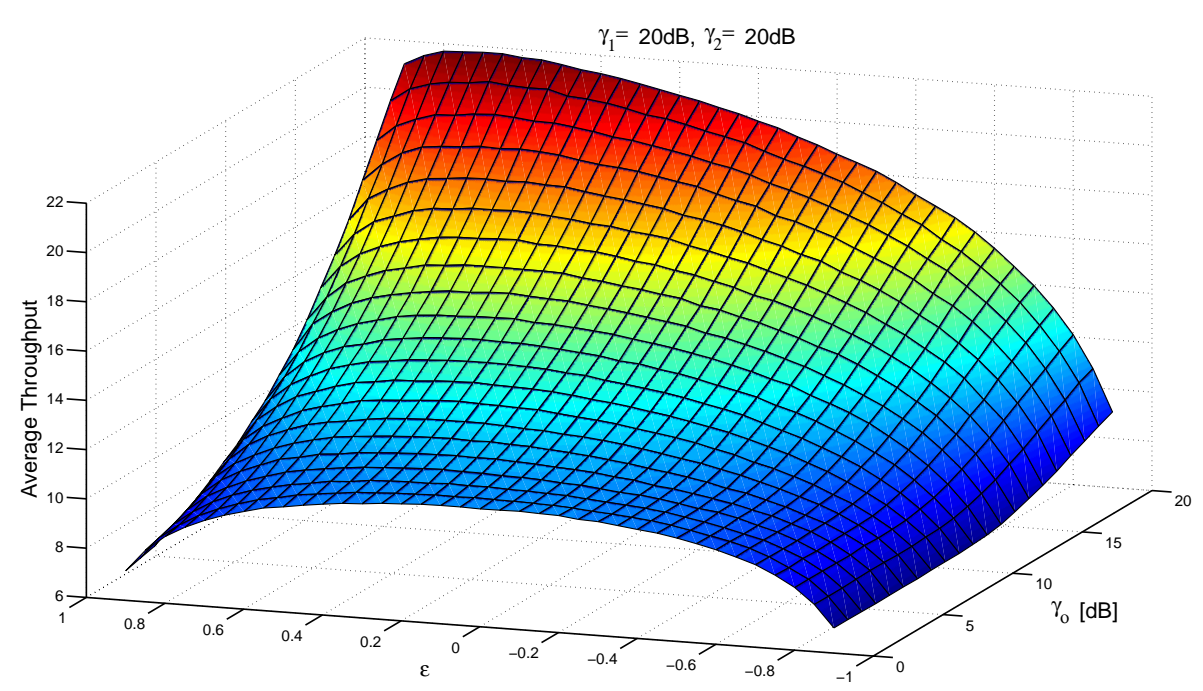

Fig. 3. Capacity (bits/symbol) versus $\gamma_{0}$ and $\epsilon$ when $\gamma_{1}=20 \mathrm{~dB}, \gamma_{2}=20 \mathrm{~dB}$, and $M=N=4$.

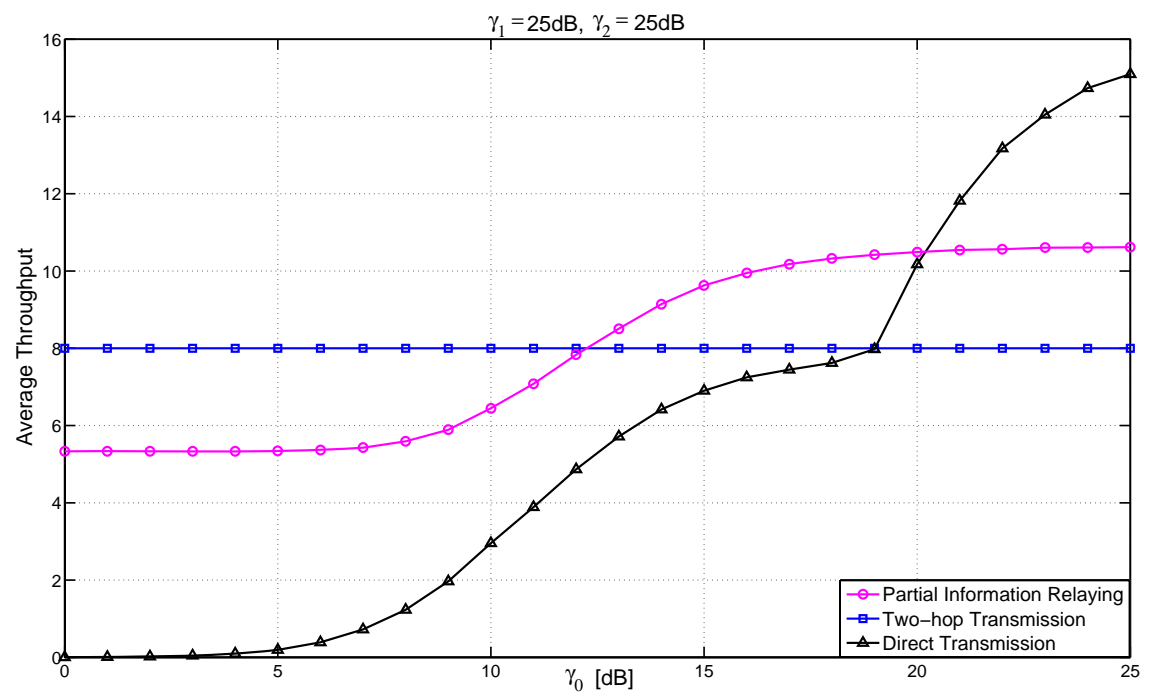

Fig. 4. Average throughput when $\gamma_{1}=25 \mathrm{~dB}, \gamma_{2}=25 \mathrm{~dB}$, and $M=N=4$.

partial information relaying. The issues of precoding/SVD that require full CSI can be addressed as a future work, in conjunction with partial information relaying for further increased gain.

\section{REFERENCES}

[1] J. Laneman, D. Tse, and G. Wornell, "Cooperative diversity in wireless networks: efficient protocols and outage behavior," IEEE Trans. Info. Theory, vol. 50, no. 12, pp. 3062-3080, Dec. 2004.

[2] A. Nosratinia and A. Hedayat, "Cooperative communication in wireless networks," IEEE Commun. Mag., vol. 42, no. 10, pp. 74-80, Oct. 2004.

[3] R. Nabar, H. Bolcskei, and F. Kneubuhler, "Fading relay channels: performance limits and space-time signal design," IEEE Journal on Sel. Areas in Commun., vol. 22, no. 6, pp. 1099-1109, Aug. 2004

[4] T. Cover and A. El Gamal, "Capacity theorems for the relay channel," IEEE Trans. Info. Theory, vol. IT-25, no. 5, pp. 572-584, Sep. 1979.

[5] M. Yuksel and E. Erkip, "Broadcast strategies for the fading relay channel," IEEE Proc. MILCOM 2004, vol. 2, pp. 1060-1065, Oct. 2004.
[6] P. Popovski and E. de Carvalho, "Improving the rates in wireless relay systems through superposition coding," IEEE Trans. Wireless Commun., vol. 7, pp. 4831-4836, Dec. 2008.

[7] A. Duel-Hallen, "Fading channel prediction for mobile radio adaptive transmission," IEEE Proceedings, vol. 95, no. 12, pp. 2299-2313, Dec. 2007.

[8] P. W. Wolniansky, G. J. Foscini, G. D. Golden, and R. A. Valenzuelar, "V-BLAST: an architecture for realizing very high data rates over the richscattering wireless channel," in Proc. of URSI International Symposium on Signals, Systems, and Electronics (ISSSE '98), pp. 295-300, Pisa, Italy, Sept. 1998.

[9] G. H. Golub and C. F. Van Loan, Matrix Computations. Johns Hopkins University Press, Baltimore, MD, 1983.

[10] S. Sfar, et. al., "Is relayed collaborative communication worth it?" in Proc. 42nd., Asilomar Conf. on Signals, Systems and Computers, pp. 146-150, 2008.

[11] Z. Lin, et. al., "MAC v. PHY: How to relay in cellular networks," in Proc. IEEE WCNC'09, pp. 1-6, 2009. 